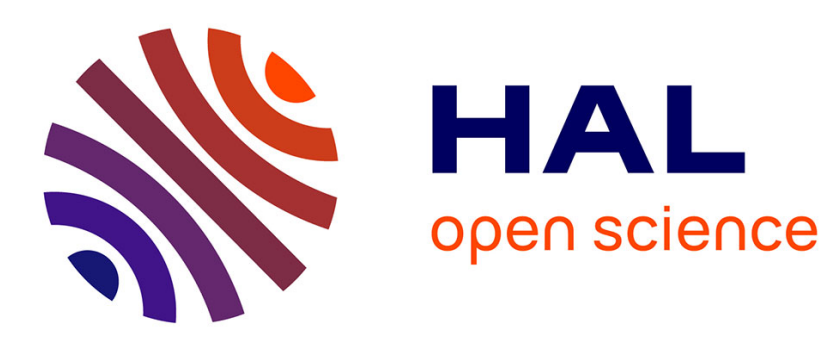

\title{
LES PROFESSIONS INTERMÉDIAIRES AU COEUR DE NOUVELLES FORMES D'ORGANISATION INDUSTRIELLE
}

\author{
Marie-Laure Buisson, Martine Gadille
}

\section{- To cite this version:}

Marie-Laure Buisson, Martine Gadille. LES PROFESSIONS INTERMÉDIAIRES AU COEUR DE NOUVELLES FORMES D'ORGANISATION INDUSTRIELLE: Enquête auprès de techniciens et d'agents de maîtrise de l'industrie aéronautique. Travail et Emploi, 2016, 4, pp.55-80. 10.4000/travailemploi.7323 . hal-02466998

\section{HAL Id: hal-02466998 \\ https://hal.science/hal-02466998}

Submitted on 4 Feb 2020

HAL is a multi-disciplinary open access archive for the deposit and dissemination of scientific research documents, whether they are published or not. The documents may come from teaching and research institutions in France or abroad, or from public or private research centers.
L'archive ouverte pluridisciplinaire HAL, est destinée au dépôt et à la diffusion de documents scientifiques de niveau recherche, publiés ou non, émanant des établissements d'enseignement et de recherche français ou étrangers, des laboratoires publics ou privés. 


\title{
LES PROFESSIONS INTERMÉDIAIRES AU CEUR DE NOUVELLES FORMES D'ORGANISATION INDUSTRIELLE
}

Enquête auprès de techniciens et d'agents de maîtrise de l'industrie aéronautique

\author{
Marie-Laure Buisson et Martine Gadille
}

DARES | « Travail et emploi »

$2016 / 4 n^{\circ} 148 \mid$ pages 55 à 80

ISSN 0224-4365

Article disponible en ligne à l'adresse :

https://www.cairn.info/revue-travail-et-emploi-2016-4-page-55.htm

Distribution électronique Cairn.info pour DARES.

(C) DARES. Tous droits réservés pour tous pays.

La reproduction ou représentation de cet article, notamment par photocopie, n'est autorisée que dans les limites des conditions générales d'utilisation du site ou, le cas échéant, des conditions générales de la licence souscrite par votre établissement. Toute autre reproduction ou représentation, en tout ou partie, sous quelque forme et de quelque manière que ce soit, est interdite sauf accord préalable et écrit de l'éditeur, en dehors des cas prévus par la législation en vigueur en France. Il est précisé que son stockage dans une base de données est également interdit. 


\section{Les professions intermédiaires au cœur de nouvelles formes d'organisation industrielle}

Enquête auprès de techniciens et d'agents de maîtrise de l'industrie aéronautique

Intermediate Categories at the Heart of New Forms of Industrial Organization. Investigation on Technicians and Supervisors in the Aircraft Industry

Marie-Laure Buisson et Martine Gadille

\section{revues.org}

Édition électronique

URL : http://travailemploi.revues.org/7323

DOI : 10.4000/travailemploi.7323

ISSN : 1775-416X

\section{Éditeur}

DARES - Ministère du travail de la solidarité et de la fonction publique

\section{Édition imprimée}

Date de publication : 1 octobre 2016

Pagination : 55-80

ISSN : 0224-4365

Distribution électronique Cairn

CAIRN INFO

CHERCHER, REPÉRER, AVANCER.

Référence électronique

Marie-Laure Buisson et Martine Gadille, « Les professions intermédiaires au cœur de nouvelles formes d'organisation industrielle », Travail et Emploi [En ligne], 148 | octobre-décembre 2016, mis en ligne le

01 octobre 2018, consulté le 24 juillet 2017. URL : http://travailemploi.revues.org/7323 ; DOI :

10.4000/travailemploi.7323 


\title{
Les professions intermédiaires au cour de nouvelles formes d'organisation industrielle
}

\author{
Enquête auprès de techniciens et d'agents de maîtrise \\ de l'industrie aéronautique
}

\author{
Marie-Laure Buisson*, Martine Gadille**
}

\begin{abstract}
Alors que la construction aéronautique s'appuie aujourd'hui sur une nouvelle forme d'organisation industrielle - l'architecture modulaire -, nous cherchons à découvrir dans cet article les conséquences de ces transformations sur les contenus du travail des techniciens et des agents de maîtrise, et sur les pratiques de gestion des ressources humaines qui leur sont appliquées. Pour ce faire, nous avons mené une enquête dans quatre entreprises du secteur en région Provence-Alpes-Côte d'Azur (un donneur d'ordre et trois soustraitants). Nos observations mettent en évidence le fait que les activités des techniciens et des agents de maîtrise ne peuvent désormais plus réellement être différenciées et, surtout, qu'elles consistent de plus en plus en de la médiation et de la coordination entre les différents services ou intervenants (ou modules) dans la chaîne de production, y compris sur les tâches de conception. Alors qu'ils doivent faire preuve de davantage d'autonomie et mettre en œuvre des compétences communicationnelles pour mener à bien leurs missions, les techniciens et les agents de maîtrise n'accèdent que rarement au statut de cadre, ce qui est source de frustration et de mécontentement à l'égard de la politique de gestion des ressources humaines menée par les entreprises.
\end{abstract}

'Institut national de la statistique et des études économiques (Insee) définit plusieurs
catégories statistiques permettant de classer les salariés : cadres et professions
intellectuelles supérieures, professions intermédiaires, employés, ouvriers, etc. Avec
la refonte de la nomenclature des « professions et catégories socioprofessionnelles »
(PCS) de 1982, apparaît la catégorie « professions intermédiaires », qui se substitue
à l'ancienne catégorie « cadres moyens ». Comme son nom l'indique, elle regroupe

\footnotetext{
* Aix-Marseille Univ., CNRS, Lest, Aix-en-Provence, France ; marie-laure.buisson@univ-amu.fr.

** Aix-Marseille Univ., CNRS, Lest, Aix-en-Provence, France ; martine.gadille@ univ-amu.fr.
} 
des professions au positionnement «intermédiaire » au sein des organisations, «à l'interface entre conception et exécution, entre cadres et opérateurs, entre entreprises, clients et fournisseurs, une position névralgique qui leur confère un rôle déterminant dans la mise en œuvre des orientations décidées par le management et dans la conduite du changement » (CADET, GUITTON, 2013, p. 20). Catégorie hétérogène, dans laquelle on retrouve de nombreuses professions (infirmière, conseiller commercial, conseiller clientèle, responsable « paie », chef d'atelier dans la construction automobile...), elle manque cependant de visibilité sociale (Bosc, 2008). Si elle a été un réel objet de recherche par le passé à partir des catégories de techniciens ou d'agents de mâ̂trise (TROuvÉ, 1997), elle constituerait aujourd'hui un «point aveugle des sciences humaines et sociales » (CADET, GuITTON, 2013, p. 20) alors même qu'elle concentre près du quart de l'emploi salarié privé (MöBus, DelanoË, 2009).

Nous nous intéressons ici aux catégories de techniciens et d'agents de maîtrise de l'industrie aéronautique ${ }^{1}$. À l'instar d'autres secteurs, la forme d'organisation industrielle s'y est redéfinie au fil du temps à la suite de l'adoption du principe de modularité par quelques constructeurs mondiaux : en amont de la production, le produit final (hélicoptère, drone) est conçu comme un assemblage de sous-ensembles cohérents technologiquement (appelés « modules »), dont non seulement l'ingénierie d'étude et de conception mais aussi la fabrication sont externalisées. La maintenance de ces sous-ensembles complexes composant les aéronefs et leurs systèmes de navigation est également externalisée (Frigant, TALBOT, 2004 ; ARAujo, 2006). Le principal donneur d'ordre est alors qualifié « d'architecte ensemblier», tandis que les soustraitants deviennent des « sous-ensembliers ». Des changements s'ensuivent dans les processus de contractualisation, d'organisation et de diversification de la relation à la sous-traitance et les clients.

Des travaux de recherche ont montré que certaines entreprises ont dans le même temps mis en œuvre une politique de rationalisation de leur chaîne de valeur, qui s'est traduite par une réduction du nombre de sous-traitants au profit des fournisseurs ayant des capacités techniques, financières et managériales pointues (HAAS, 2010). Ils ont mis en évidence que dans les chaînes de sous-traitance, les catégories de techniciens et d'agents de maîtrise se retrouvent au cœur de processus organisationnels de transformation des fonctions support, certification, suivi de maintenance, contrôle et gestion (qualité, coûts, délais) (GÉLOT, 2006 ; HAAS, 2010). De telles évolutions avaient déjà été relevées, mais pour les seuls agents de maîtrise (Trouvé, 1997). Dans cet article, nous montrons que si les catégories d'ingénieurs et de cadres se concentrent dans les services de recherche et développement (R\&D) interne et dans les entreprises soustraitant des services numériques, les professions intermédiaires de techniciens et agents de maîtrise sont au centre des processus d'innovation. Ces derniers traduisent de fait les contraintes issues des services d'étude, de conception et de méthodes, et aussi du

1. Nous avons mené une enquête de terrain dans quatre entreprises spécialisées dans la production de sous-ensembles et de composants pour hélicoptères (encadré 1 , infra). 
prototypage, de la fabrication, de la maintenance, de la commercialisation ou de la normalisation (HATCHUEL, 1996).

Nous choisissons d'observer ici les techniciens et agents de maîtrise de façon parallèle. Ces deux sous-catégories de la PCS « Professions intermédiaires », bien que très différents à la fois en termes de niveau de diplômes, d'origine sociale, de parcours professionnels (Trouvé, 1997), ou encore de sentiment d'appartenance (DEAUVIEAU, Dumoulin, 2010), semblent vivre, dans l'aéronautique, des évolutions communes qui les conduisent à jouer un rôle similaire de médiation dans l'innovation, que nous précisons par la suite.

Dans une première partie, nous revenons sur la définition et la caractérisation statistique des catégories de techniciens et d'agents de maîtrise. Puis nous étudions les transformations industrielles à l'œuvre dans l'industrie aéronautique et leur impact sur ces deux catégories intermédiaires, en accordant une importance particulière aux conséquences organisationnelles et technologiques entraînées par la mise en application du principe de modularité. Dans une deuxième partie, nous présentons la méthodologie de l'enquête que nous avons menée puis, dans une troisième et dernière partie, nous exposons les principaux résultats et leur implication pour l'analyse plus globale des professions intermédiaires.

\section{Professions intermédiaires et transformations industrielles dans l'aéronautique : revue de la littérature}

Nous rappelons d'abord les enjeux socio-économiques que posent les professions intermédiaires, et plus particulièrement les catégories de techniciens et d'agents de maîtrise, tels qu'appréhendés dans la littérature.

\section{Une approche sociétale et statistique des catégories de techniciens et d'agents de maîtrise}

En 1992, dans la continuité des travaux de Géraldine de BonnAFos (1989), Annette JobERT et Michèle TALLARD remarquent que les techniciens constituent une catégorie intermédiaire à la définition imprécise en dépit de sa place dans le champ productif. En effet, différents facteurs liés aux classifications de branche et aux formes de gestion de la main-d'œuvre se combinent pour créer des sources de tension dans la relation salariale qui les caractérisent. Dans leur recherche, François EyRAud, Alain D'IRIBARNE et Marc MAURICE (1988) étudient notamment la transformation des activités des opérateurs : soumises à des contraintes productives de délais, de qualité et d'introduction de nouvelles technologies, elles s'orientent de plus en plus vers l'analyse, le contrôle et la maintenance. Néanmoins, l'autonomie des techniciens dans les tâches de programmation n'est pas pleinement reconnue. L'agent de maîtrise, quant à lui, se voit confier un rôle d'animation. 
De tels constats s'inscrivent dans la continuité de ce qu'ont montré les travaux fondateurs de ce courant de travaux : ils mettent en évidence une forme sociétale de stratification sociale des qualifications ${ }^{2}$ définissant des modes de mobilité et de gestion des carrières (MAURICE et al. 1982). Tandis que la force de l'ouvrier qualifié allemand (Facharbeiter) et la rareté de l'encadrement moyen (Foremann) dans l'entreprise allemande étaient associées aux caractéristiques du système éducatif et de formation dans lequel les diplômes de formation professionnelle de base tenaient une large part ; au contraire, dans le cas français, la faiblesse de la formation professionnelle au sein du système éducatif était cohérente avec une qualification ouvrière de faible niveau et un poids d'autant plus important de la maîtrise et de l'encadrement moyen. Au début des années 1990, certains observateurs craignent toutefois un avenir teinté de tensions pour cette catégorie :

«L'avenir de la catégorie dépend aussi du type de segmentation qui résultera de l'application des règles. Si les passages à la catégorie ingénieur restent exceptionnels et si les embauches continuent à s'effectuer majoritairement au niveau bac +2 , on risque de s'orienter vers une catégorie relativement homogène mais dont les aspirations en termes de contenus de travail et de déroulement de carrière resteraient insatisfaites. »

(JoBERT, TALLARD, 1992, p. 155)

D'un point de vue statistique, la catégorie des techniciens et des agents de maitrise peut être décrite comme suit. Actuellement, le tiers des effectifs des professions intermédiaires des entreprises françaises est classé en techniciens, contre $14 \%$ en agents de maîtrise (MöBus, DelanoË, 2013).

Chez les techniciens, les niveaux de formation sont hétérogènes (MöBus, Delanö̈, 2009). Tous âges confondus, on y trouve $30 \%$ de niveaux III, $23 \%$ de niveaux IV et $26 \%$ de niveaux V. Pour les techniciens de moins de 30 ans, les proportions ne sont pas les mêmes : $50 \%$ ont une formation de niveau III (BTS ou DUT ${ }^{3}$ ) tandis que $15 \%$ ont un baccalauréat professionnel (niveau IV) et seulement $10 \%$ ont un niveau $\mathrm{V}$. Cette différence est en partie expliquée par des flux de sortie précoces, mais aussi par les flux d'entrée dans la catégorie qui se caractérisent par la persistance d'une mobilité ascendante issue de salariés ayant un statut d'ouvrier ou d'employé, dont certains ont un diplôme de niveau bac +2 . Près de $40 \%$ des techniciens travaillent dans la fabrication industrielle - ils y occupent des emplois relevant des services études, méthodes, $R \& D$ ou production -, ce qui en fait la fonction la plus représentée. Dans l'industrie aéronautique, leur répartition entre les niveaux V, IV et III est quasi équivalente, tous âges confondus (MöBus, DELANOË, 2009).

2. La qualification est ici entendue comme une validation collective de connaissances déclaratives et procédurales (DE Terssac, Chabaud, 1990 ; cf. infra). Elle se traduit par l'obtention d'un diplôme, d'une certification et/ou d'un niveau d'affectation de l'individu dans une grille de classification indiciaire des emplois déterminée par une convention collective. Cette reconnaissance est à la fois un mode de construction du savoir et l'expression d'un rapport social qui dépasse l'entreprise et implique le système éducatif et de relations professionnelles (MAURICE et al., 1982).

3. Brevet de technicien supérieur ; diplôme universitaire de technologie. 
Contrairement aux techniciens, les profils de formation des agents de maitrise sont plutôt homogènes : la proportion des diplômés de niveau $\mathrm{V}$ est importante (45 \%) alors que les niveaux III représentent $14 \%$ des effectifs. Au cours des vingt dernières années, le niveau $\mathrm{V}$ a connu une augmentation et s'est substitué aux non-diplômés. La structure d'âge de la catégorie semble confirmer que les personnes expérimentées ont une mobilité verticale conséquente. Ces caractéristiques nationales des agents de maîtrise ne se retrouvent pas sur notre terrain, où les agents de maîtrise que nous avons rencontrés sont nettement plus diplômés.

Observons à présent plus précisément les transformations à l'œuvre dans l'industrie aéronautique pour mieux cerner les facteurs organisationnels d'évolution dans ce secteur.

\section{Principe de modularité et entreprises pluri-technologiques dans l'aéronautique}

À l'heure actuelle, dans le secteur aéronautique, l'organisation industrielle évolue sous l'effet des modifications de stratégies des principaux constructeurs mondiaux. D'une part, ces derniers tentent de partager la prise de risque en termes de recherche et développement en formant des partenariats. D'autre part, ils font le choix de réorganiser la sous-traitance, qui doit non seulement obéir à des normes drastiques de qualité et de délais mais aussi être en capacité de s'adapter aux innovations de procédés et de matériaux.

En R\&D, Virginia ACHA et Stefano BRUSONI (2008) soulignent l'importance croissante de l'avionique (c'est-à-dire l'environnement informatique et digital permettant la conduite et la régulation d'ensemble du système volant). Cette évolution, qui suppose un renouvellement en profondeur des savoirs, s'impose dans la mécanique « classique » et contribue à déstructurer les frontières de l'industrie. Ajoutons qu'à la différence de l'avion "classique », la révolution digitale affecte également les usages potentiels de l'hélicoptère (ou du drone). Les dispositifs informatiques et optiques embarqués sont installés sur de nouveaux types d'aéronefs et mis au service de nouvelles tâches telles que la surveillance, la cartographie prévisionnelle de feux de forêts, le diagnostic d'ouvrages d'arts, etc. Là aussi, une recomposition des savoirs est à l'œuvre. Des entreprises mobilisant plusieurs technologies ont donc émergé ; leur avantage concurrentiel repose sur leur capacité à opérer des transferts (et des recompositions) de savoirs d'un champ d'activité à un autre.

En ce qui concerne la sous-traitance, les évolutions dans l'aéronautique semblent proches de celles de l'automobile. Elles se traduisent, comme nous l'avons dit, par la mise en place de la modularité (BRUSONI et al., 2001). Les produits, devenus de plus en plus complexes, sont décomposés en modules (sous-systèmes) dont chacun est décrit dans un cahier des charges. L'architecte ensemblier s'assure que ces modules s'assemblent correctement les uns avec les autres. Leurs interfaces sont « découplées »; autrement dit, la modification de l'un des sous-ensembles n'affecte pas a priori un autre sous-ensemble. Les travaux de recherche effectués sur la modularité soulignent 
qu'elle implique des enjeux à la fois technologiques et organisationnels. C'est parce que l'architecte maîtrise les relations entre les sous-systèmes qu'il peut segmenter la conception et la réalisation des modules, soit en interne (on parle alors d'entreprise intégrée), soit en externe. Dans ce dernier cas, la réorganisation de la sous-traitance favorise l'apparition, en nombre restreint, de concepteurs-ensembliers de sous-systèmes, qui eux-mêmes délèguent et sous-traitent des sous-ensembles. Modularité technologique et organisationnelle sont donc inséparables et vont, selon certains auteurs, de pair avec une redéfinition des compétences (BRUSONI et al., 2001), l'entreprise architecte conservant un « surplus » de compétences en raison de son rôle d'intégrateur. Les compétences technologiques et organisationnelles dont parlent S. Brusoni et ses coauteurs s'appuient sur des connaissances à la fois conceptuelles car de type académique, et informelles car acquises par l'expérience. Cette approche rejoint celle de G. De TerssaC et C. Chabaud, (1990, p. 127), citant J. Leplat (1988), qui définissent la compétence comme la combinaison de trois types de connaissances : les connaissances générales dites déclaratives (issues principalement de l'enseignement initial et/ou de la formation continue) ; les connaissances opératives dites procédurales «tournées vers la réalisation d'un but et acquises par la pratique » à partir de la mobilisation des connaissances déclaratives dans des contextes particuliers ; et enfin, les connaissances routinières. Si ces dernières s'acquièrent également par la pratique, l'adjectif qui les qualifie suggère qu'elles sont mises en œuvre sans contrôle conscient du sujet.

Amorcée dès les années 2000 dans l'industrie aéronautique, systématisée par exemple avec l'Airbus A 380, l'intégration modulaire repose aussi sur le partage de normes de certification aéronautique, qui doivent être prises en compte tout au long de la chaîne de production. Elle a recours à des outils d'échanges d'informations qui permettent de se passer de la proximité géographique et de réintroduire ainsi des contraintes « pures » de marché en favorisant la concurrence entre «sous-ensembliers »; cette thèse fait néanmoins l'objet de débats (ARAUjo, 2006).

Nous faisons l'hypothèse que ces transformations chez les constructeurs aéronautiques et leurs sous-traitants ont des effets sur la catégorie des techniciens et des agents de maîtrise. D'un point de vue analytique, nous nous situons dans le prolongement des observations réalisées dans le courant des années 1980 et 1990, mais empruntons une entrée liée à la transformation de la division sociale du travail entre entreprises dans les territoires (GADILLE, MACHADO, 2010, 2012).

\section{Techniciens et agents de maîtrise au cœur des savoirs d'innovation}

Dans un contexte d'innovation intensive, la manière dont sont pensés les métiers et les missions des « acteurs et des activités de conception » renouvelle des situations de tension dans les façons d'apprendre et de coopérer au travail (HATCHUEL et al., 2002, p. 30). Coopérer implique des prescriptions croisées consistant en échanges réciproques de connaissances, en particulier expérientielles, indépendamment des positions hiérarchiques ou des positions d'autorité (HATCHUEL, 1996). Nous supposons 
que les techniciens et agents de maîtrise, en qualité de professions intermédiaires, sont plus particulièrement touchés par cette nouvelle vague de rationalisation, à la fois dans l'entreprise mais aussi dans ses réseaux de sous-traitance.

Cependant, ces transformations interviennent dans un contexte sociétal où persiste une stratification sociale « à la française ». Dans la mesure où la technique y est assimilée à une science et non à un savoir-faire empirique, l'ingénieur se trouve doté d'un statut social élevé et peu accessible. Sa position prépondérante en matière de prise de décision est source de cloisonnements entre services de conception, de méthodes, de prototypage et de fabrication, non seulement au sein d'une entreprise donnée mais aussi entre une entreprise cliente et ses fournisseurs (MAURICE, Sorge, 1989). Ce type de relations intra- et inter-entreprises correspond à une forme d'industrialisation conçue en rupture avec le modèle artisanal, car elle privilégie les savoirs de conception sur ceux de fabrication.

L'innovation, quelle que soit sa nature (technologique, liée aux procédés ou aux produits et aux services ${ }^{4}$ ), nécessite la résolution de problèmes. Celle-ci a donc tendance à être confiée aux niveaux intermédiaires, qui occupent des positions charnières dans la production de connaissances expérientielles et dans la validation sociale des savoirs acquis au cours de l'activité (BERCOT et al., 1988 ; WiTTORSKI, 1998). C'est ainsi que, dans le cadre des modernisations industrielles, les techniciens et agents de maîtrise sont fortement sollicités pour finaliser processus et produits, services et procédés innovants (TROUVÉ, 1997). Les responsabilités qu'ils assument (span of control) sur les activités techniques sont plus importantes ; le travail indirect (planification, normalisation, conception, médiation) qu'ils effectuent est en augmentation ; les rapports d'autorité s'appuyant sur des relations hiérarchiques strictes sont relâchés. Aussi, les relations qu'entretiennent et les techniciens et les agents de maîtrise avec les services de conception et d'ingénierie d'un côté, et les sous-traitants ou donneurs d'ordre de l'autre, ont évolué. Si ces professions ont réussi à s'adapter au changement, c'est parce que, tout en ayant suivi des formations techniques et/ou théoriques, elles ont également fait preuve de capacité à apprendre ou à s'auto-former (CARRÉ et al., 1997) et à valider dans la coopération, donc dans l'activité, un savoir toujours renouvelé.

Dès lors, techniciens et agents de maîtrise aspirent davantage qu'auparavant à une mobilité ascendante vers des positions professionnelles plus valorisées, telles que celles des ingénieurs et des cadres (BOUFFARTIGUE, 1994). Les départs à la retraite des anciens agents de maîtrise ou des techniciens qui avaient acquis une expertise individuelle précieuse ou qui constituaient de petites équipes très compétentes, combinés à la mobilité ascendante des jeunes techniciens, engendreraient une déperdition des savoirs de l'entreprise. Cependant, comme par le passé, ces possibilités de mobilité vers le statut d'ingénieur restent encore aujourd'hui très faibles (BOUFFARTIGUE, GADÉA, 1996) ; elles s'expliquent notamment par la non-reconnaissance du niveau de licence

4. Essais, tests, pilotage, suivi logistique, maintenance, réparation, prototypage/usinage. 
professionnelle dans la convention collective de l'Union des industries et métiers de la métallurgie (UIMM).

En résumé, le renouvellement des contenus du travail des techniciens et des agents de maîtrise a entraîné de nouvelles contraintes d'apprentissage et de validation de ces apprentissages dans l'activité de coopération tout au long de la chaîne de valeur. Ces catégories, à la composition encore hétérogène, expérimentent de nouveaux rapports au travail, à la formation et à la carrière ; autant de pratiques et d'aspirations que les entreprises ne sont pas forcément en mesure d'intégrer dans leur gestion des ressources humaines (GRH).

\section{Méthodologie de l'enquête qualitative}

Nous présentons d'abord le secteur aéronautique sur lequel a porté l'enquête, puis la méthodologie employée pour observer l'impact des transformations industrielles qu'il a connues sur les techniciens et les agents de maitrise, à partir des contenus de travail et du management des ressources humaines associé.

\section{Présentation du secteur de l'industrie aéronautique}

La construction aéronautique regroupe les métiers de la conception, de la fabrication et de la commercialisation des aéronefs, tels les avions ou les hélicoptères. Elle fait partie des activités du secteur aéronautique et spatial. Composée de près de 4000 entreprises en France, la filière aéronautique emploie plus de 320000 personnes en 2015 , pour un chiffre d'affaires de 49 milliards d'euros ${ }^{5}$. Elle génère également de l'activité et des emplois dans les entreprises sous-traitantes qui peuvent relever d'autres secteurs comme, celui des industries d'équipement mécanique (chaudronnerie par exemple) ou des équipements électriques et électroniques. Au total, plus de 310000 emplois sont ainsi concernés en 2012, en comptant les sous-traitants.

Depuis le début des années 2000, le secteur est dans une phase charnière. Des projets de long terme associés à une logistique de type supply chain management ${ }^{6}$ (gestion de la chaîne des approvisionnements) l'obligent à anticiper l'évolution des effectifs. De plus, depuis 2010, le secteur doit faire face à une croissance record de son activité, qui se traduit par des carnets de commandes représentant jusqu'à sept ou huit années de production.

5. Direction générale des entreprises, « La filière aéronautique »; en ligne : http://www.entreprises.gouv.fr/conseilnational-industrie/la-filiere-aeronautique, consulté le 22 mai 2017.

6. La logistique de type supply chain management a pour objectif d'améliorer les résultats de l'ensemble de la chaîne d'approvisionnement des fournisseurs, tant du point de vue de la complétude du service rendu, de sa qualité que de son coût. Pour ce faire, elle a recours à de nombreux outils, informatiques en particulier (logiciels de planification, de fabrication, d'optimisation des stocks, etc.). Le supply chain management a donc transformé et élargi les compétences en logistique au-delà de la seule gestion des stocks. 
Selon l'Insee, les métiers ou spécialités les plus recherchés sont les ajusteurs, les soudeurs, les chaudronniers et les opérateurs. Comme le souligne le Groupement des industries françaises aéronautiques et spatiales (Gifas), « les entreprises évoquent de fortes difficultés à trouver des profils qualifiés, dotés d'une expérience dans le monde de l'aéronautique. De plus, le manque d'attractivité des filières scolaires techniques, voire scientifiques, et du secteur de l'industrie accentue les tensions en recrutement ${ }^{7}$ ». Les entreprises du secteur doivent donc élargir leurs critères de recrutement et mettre en place une véritable politique de formation et d'intégration. La tendance serait donc de miser sur des formations « maison » post-recrutement.

\section{Terrain et données}

Pour observer les modifications dans les contenus du travail des techniciens et des agents de maîtrise, et les pratiques de GRH qui en découlent, nous avons enquêté en région Provence-Alpes-Côte d'Azur (Paca) où l'industrie aéronautique et spatiale est particulièrement présente : l'un des leaders mondiaux du secteur y est implanté, de même qu'un fabricant d'équipements spatiaux et deux centres d'essais en vol. La principale entreprise, Aéro ${ }^{8}$, appartient à un groupe multinational ; elle contrôle par ailleurs un réseau de sous-traitants de proximité, avec lesquels elle entretient des rapports de dépendance plus ou moins étroits et dont elle constitue le donneur d'ordre (DO) principal. Notre étude porte donc sur un bassin d'emploi localisé.

Pour repérer et découvrir ce que recouvrent les emplois de catégorie intermédiaire de l'industrie aéronautique, nous avons utilisé les fiches emplois éditées par l'espace orientation de l'association Airemploi ${ }^{9}$; nous avons ensuite comparé les informations ainsi obtenues à celles que nous avons collectées sur notre terrain (encadré 1).

À partir de notre panel de cas, nous avons étudié quatre entreprises de tailles différentes (de 100 à 9000 salariés) : Aéro et trois de ses sous-traitants. Pour chacune de ces entreprises, nous avons interviewé des membres de l'équipe dirigeante lors de la première vague de recherche : directeur industriel, directeur des $\mathrm{RH}$, responsable des relations sociales, mais aussi des techniciens et des agents de maîtrise (tableau). Nous avons également mené des entretiens avec les représentants des principaux syndicats $\left(\mathrm{CFDT}\right.$ et $\mathrm{FO}^{10}$ ) d'Aéro afin d'avoir leur point de vue concernant la gestion des carrières, le recrutement, la formation des techniciens et des agents de maitrise. Cette première phase avait pour objectif d'obtenir une présentation de l'entreprise, de

\footnotetext{
7. Cité par Roy G. (2013), «L'aéronautique, ce secteur qui embauche à tour de bras », L'Express - L'Expansion ; en ligne : http://lexpansion.lexpress.fr/entreprises/l-aeronautique-ce-secteur-qui-embauche-a-tour-de-bras_1332619.html, consulté le 22 mai 2017.

8. Les noms des entreprises ont été modifiés.

9. L'association Airemploi a été créée en 1999 à l'initiative du groupe Air France, de la Fédération nationale de l'aviation marchande (Fnam) et du Groupement des industries françaises aéronautiques et spatiales (Gifas), en partenariat avec l'Armée de l'air et Aéroports de Paris. Elle met à disposition des fiches emplois validées par les instances collectives de l'aéronautique.

10. Confédération française démocratique du travail ; Force ouvrière.
} 


\section{ENCADRÉ 1}

\section{Présentation de l'enquête}

Les observations mobilisées dans cet article ont été réalisées au cours de trois vagues de recherche effectuées entre 2008 et 2012 sur un même pôle de compétitivité aéronautique et spatial en région Paca.

Lors de la première vague qui a eu lieu entre janvier et octobre 2008, l'équipe de recherche a mené des entretiens qualitatifs semi-ouverts auprès de responsables d'entreprises, de responsables syndicaux et d'agents de l'inspection du travail. Nous avons donc rencontré : 29 responsables d'entreprises (3 grandes entreprises DO, dont l'entreprise Aéro, $19 \mathrm{PME}^{1}$ aux effectifs inférieurs à 100 salariés, 7 entreprises aux effectifs compris entre 110 et 500 salariés), 2 représentants syndicaux (issus du comité d'entreprise du principal donneur d'ordre) et 2 agents des services de l'inspection et de la direction régionale du travail représentant l'État en région.

La deuxième vague d'observations, entre septembre 2008 et janvier 2011, provient d'une recherche action conduite dans ce même pôle. Elle visait à évaluer, en cours de process, une action collective de transfert interne des connaissances entre salariés dans 10 PME sous-traitantes, impliquant des techniciens, des agents de maîtrise et des entrepreneurs (GADILlE, MACHADO, 2012).

La dernière vague d'observation, que nous avons réalisée de manière conjointe entre mai 2009 et janvier 2012, a consisté en des entretiens uniquement avec des techniciens et des agents de maîtrise d'entreprises déjà étudiées dans les phases précédentes. Ils nous ont permis de contribuer à une recherche collective récente sur les catégories intermédiaires (CAdet, Guitton, 2013 ; Buisson, Gadille, 2013).

1. Petites et moyennes entreprises.

découvrir les réformes en cours et d'accéder à des documents tels que le bilan social, la déclaration $2483^{11}$, les principaux textes et accords d'entreprise concernant la gestion interne de l'emploi, les supports de communication interne, etc.

Nous avons ensuite mené 23 entretiens semi-directifs d'une durée comprise entre 45 minutes et 3 heures avec des techniciens et des agents de maîtrise. Nous avons cherché à rencontrer des travailleurs aux situations contrastées du point de vue de l'âge, du sexe, du diplôme, de la fonction et du contenu d'activité (tableau).

Pour réaliser nos entretiens, nous avons construit deux guides : RH et dirigeants d'une part ; techniciens et agents de maîtrise d'autre part (encadré 2 pour ce dernier). Nous nous sommes particulièrement intéressées au contenu de l'activité des techniciens et des agents de maitrise dans l' aéronautique et aux modes de gestion du personnel qui

11. Supprimée en mars 2014, la déclaration 2483 s'appliquait aux entreprises de 10 salariés et plus, qui devaient justifier auprès de l'administration l'utilisation de leur budget formation. Ce faisant, elles rendaient compte de leur participation au développement de la formation professionnelle continue. 


\section{ENCADRÉ 2}

\section{Guide d'entretien à destination des techniciens et des agents de maîtrise}

Récolte des données biographiques de base pour les deuxième et troisième vagues de recherche :

Diplômes, différents emplois occupés au cours de la carrière, ancienneté dans l'entreprise, différentes fonctions occupées dans l'entreprise, qualification, statut d'emploi.

\section{Partie 1 : travail et position dans l'entreprise}

Quelle est votre activité ? Les missions qui vous sont confiées ? Vos responsabilités ?

Quelles sont les compétences qui vous apparaissent les plus importantes dans votre activité ?

Comment évolue[nt] votre activité, vos compétences et votre place au sein de l'organisation du travail ?

Comment votre activité est-elle formalisée ?

Vous estimez-vous bien payé pour votre travail?

Y a-t-il pour vous un élément fédérateur dominant qui caractérise votre catégorie?

\section{Partie 2 : recrutement et formation}

Par quelle voie êtes-vous arrivé sur ce poste?

Comment s'est passée votre intégration?

Quel est votre usage de la formation continue?

Comment se transmettent les compétences dans votre entreprise?

\section{Partie 3 : Gestion du parcours professionnel}

Quelle perception avez-vous de votre parcours?

Comment avez-vous évolué ?

Quels sont vos projets?

leur sont appliqués. Ces entretiens ont été retranscrits et ont fait l'objet d'une analyse thématique « semi-formatée » (ROMELAER, 2005).

Chez Aéro, le donneur d'ordre, les techniciens sont souvent diplômés de niveau III ou détenteurs d'une licence 3 , et ont obtenu une promotion intervenue sans changement ni de qualification ni de la définition de leur poste, bien que celle-ci s'éloigne d'un contenu réel qui évolue très vite avec l'expérience. Les agents de maîtrise rencontrés, plus anciens, n'exercent pas plus de responsabilités d'encadrement que les techniciens. Par contre, chez les sous-traitants, les agents de maîtrise sont parfois des personnes qui, devenues responsables d'atelier par exemple, encadrent des techniciens. En somme, chez le donneur d'ordre étudié ici, techniciens et agents de maîtrise ont les mêmes fonctions et activités, même si elles sont évolutives, alors que chez les sous-traitants, l'appartenance à l'une ou l'autre catégorie correspond davantage à des niveaux de responsabilités différents. Chez le donneur d'ordre, les activités soustraitées étant très nombreuses, les professions intermédiaires semblent jouer un rôle 


\section{TABLEAU - Liste des entretiens réalisés}

\begin{tabular}{|c|c|c|c|c|c|}
\hline Entreprise & Niveau, prénom & Intitulé du poste & Sexe & Diplôme & Âge \\
\hline \multicolumn{6}{|c|}{$\begin{array}{l}\text { AÉRO } \\
\text { Environ } 9000 \text { salariés pour l'établissement concerné } \\
\text { Activité : construction aéronautique }\end{array}$} \\
\hline & \multicolumn{5}{|l|}{$\begin{array}{l}\text { Responsable relations sociales, } \\
\text { Jacques }\end{array}$} \\
\hline & \multicolumn{5}{|l|}{ Représentant FO, Pierre } \\
\hline & \multicolumn{5}{|l|}{ Représentant CFDT, Pascal } \\
\hline & Technicien, Alexandre & Préparateur méthodes & Homme & $\begin{array}{c}\text { Licence } 3 \\
\text { professionnelle }\end{array}$ & $\begin{array}{l}\text { Moins de } \\
25 \text { ans }\end{array}$ \\
\hline & Technicien, Christelle & $\begin{array}{l}\text { Animateur pièces } \\
\text { manquantes }\end{array}$ & Femme & Baccalauréat & $35-45$ ans \\
\hline & Technicien, Michael & Préparateur méthodes & Homme & Licence 3 pro & $25-35$ ans \\
\hline & Technicien, Nicolas & $\begin{array}{l}\text { Technicien support } \\
\text { client }\end{array}$ & Homme & Licence 3 pro & $25-35$ ans \\
\hline & Technicien, Mathieu & Technicien navigabilité & Homme & BTS & $25-35$ ans \\
\hline & Technicien, Carole & Technicien logistique & Femme & DUT & $25-35$ ans \\
\hline & Technicien, Aurélien & Technicien essai au sol & Homme & Licence 3 & $25-35$ ans \\
\hline & Agent de maîtrise, Jean-Marc & Préparateur méthodes & Homme & CAP & $55-65$ ans \\
\hline & Agent de maîtrise, Michel & Technicien prototypage & Homme & CAP & $55-65$ ans \\
\hline
\end{tabular}

\section{PROTOMÉTAL}

230 salariés

Activité : mécanique industrielle, site de fabrication d'une multinationale écossaise

\begin{tabular}{|c|c|c|c|c|}
\hline DRH, Loïc & & & & \\
\hline \multicolumn{5}{|l|}{ Directeur industriel, Guy } \\
\hline Technicien, Maxime & Préparateur méthodes & Homme & BTS & $25-35$ ans \\
\hline Technicien, Frédéric & $\begin{array}{l}\text { Opérateur de machine- } \\
\text { outil à commande } \\
\text { numérique }(\mathrm{MOCN})\end{array}$ & Homme & CAP & $25-35$ ans \\
\hline Agent de maîtrise, Christophe & $\begin{array}{l}\text { Responsable } \\
\text { programmation }\end{array}$ & Homme & BTS & $35-45$ ans \\
\hline
\end{tabular}

\section{SURFACHIMIE}

200 salariés

Activité : traitement et revêtement des matériaux, chimie, entreprise régionale

\begin{tabular}{|c|c|c|c|c|}
\hline Directeur général, Bernard & & & & \\
\hline \multicolumn{5}{|l|}{ DRH, Thomas } \\
\hline Agent de maîtrise, Dominique & $\begin{array}{l}\text { Responsable } \\
\text { conformité }\end{array}$ & Femme & Licence 3 & $55-65$ ans \\
\hline
\end{tabular}

\section{USIREVA}

100 salariés

Activité : mécanique industrielle, filiale d'un grand groupe

\begin{tabular}{|c|c|c|c|c|}
\hline DRH, Laurent & & & & \\
\hline Technicien, Jean & Préparateur méthodes & Homme & CAP & $25-35$ ans \\
\hline Agent de maîtrise, Luc & Chef d'atelier & Homme & DUT & $25-35$ ans \\
\hline
\end{tabular}

* Certificat d'aptitude professionnelle. 
de concepteur-prescripteur technique, logistique et normatif vis-à-vis d'entreprises sous-traitantes diverses, dont chacune a une spécialité bien particulière et circonscrite, ce qui expliquerait les différences d'attributions des professions intermédiaires entre donneur d'ordre et sous-traitants.

Toutes entreprises confondues, les agents de maîtrise sont souvent plus âgés que les techniciens, mais sans nécessairement être plus diplômés : l'ancienneté serait donc l'un des facteurs permettant de monter dans la hiérarchie et en responsabilité.

\section{Résultats : une activité de coordination interne et externe peu reconnue et encadrée}

Notre objectif est à présent de découvrir ce qui peut différencier ou rapprocher les techniciens et agents de maîtrise en termes de contenu du travail et d'examiner comment leur rôle évolue dans la nouvelle configuration qui structure le secteur aéronautique.

\section{Vécu au travail des techniciens et agents de maîtrise dans l'industrie aéronautique}

Les fonctions occupées par les techniciens et agents de maîtrise des entreprises enquêtées sont très diverses : préparateur méthodes, technicien logistique et approvisionnement, technicien support client, technicien en ingénierie de maintenance, technicien d'essais, etc. Bien souvent, les techniciens ne travaillent plus en atelier ; leurs tâches relèvent davantage du contrôle qualité, de la normalisation et du management dans la supply chain. Pour la plupart, ils ne font pas d'usinage et n'encadrent pas d'équipes, ce qui n'exclut pas qu'ils aient des responsabilités sur l'activité d'autres services, internes ou externes (sous-traitants).

Les missions confiées aux techniciens sont, logiquement et en priorité, d'ordre technique :

«Le bureau d'études fournit un plan qui est inexploitable par les compagnons, donc nous, on l'interprète, on fait les fiches techniques (plans de montage, consignes d'entretien, etc.), on fait fabriquer les pièces et on s'occupe aussi de l'approvisionnement. Je fais donc de la maintenance et la réparation sur les appareils, anciens comme nouveaux, et du retrofit ${ }^{12}$.»

(Alexandre, Aéro, licence 3 Pro, moins de 25 ans)

L'exercice du métier implique également de respecter les règles et les normes de sécurité : les techniciens doivent pour cela connaître les machines de façon approfondie,

12. Le retrofit désigne le remplacement de composants anciens ou obsolètes par de plus récents sans modification de fonction. 
maîtriser le processus de fabrication parfois complexe et mobiliser la documentation associée :

« Sur la machine, on a une responsabilité en termes de sécurité du pilote notamment, on certifie que la machine marche bien [...]. Je m'occupe de tout ce qui est navigabilité de l'appareil, je vais sur les sites de motoristes, je reçois des consignes et de l'information de tout ce qui est groupement pour la sécurité de l'aviation civile (GSAC), je prends toutes les infos du GSAC et je diffuse les impératifs : je les envoie aux opérateurs au pied des machines ; ces informations remontent du réseau. Je travaille avec des services différents pour créer des bulletins, qui permettent de modifier la machine et de résoudre les problèmes. Il y a les impératifs recommandés et ceux qui sont facultatifs. En fait, je fais de la veille et de l'information sur les normes et la législation pour la navigabilité. »

(Mathieu, Aéro, BTS, 25-35 ans)

Les changements du métier concernent essentiellement les aspects techniques, du fait de l'évolution des technologies et des certifications et normes associées : « Depuis deux ans, ça fait six fois que je change de procédure. À chaque fois je refais le boulot, ça n'a plus rien à voir » (Mathieu, Aéro, homme, BTS, 25-35 ans). Outre la réalisation des fiches techniques, les techniciens que nous avons rencontrés insistent sur les activités de coordination qu'ils effectuent : ils supervisent en effet étroitement la fabrication des pièces et la gestion de l'approvisionnement. Ils doivent donc faire preuve de capacités d'écoute et de communication, notamment lorsqu'ils sont préparateurs méthodes et jouent un rôle d'intermédiaire entre savoirs de différentes natures. Or la coordination n'est pas mentionnée dans les fiches de poste : elle dépasse donc les attendus.

Les agents de maîtrise ont des missions très proches de celles des techniciens. Ainsi, Christophe, responsable programmation encadrant des techniciens pilotes de MOCN à ultra-haute vitesse pour pièces prototypes, indique :

« Je reçois une pièce, je vais réfléchir sur quelle machine, combien de phases, la taille du brut, les outils que je vais utiliser. Après le programmeur transcrit le parcours d'outils. »

(Christophe, Protométal, BTS, 35-45 ans)

Chez le donneur d'ordre, les agents de maîtrise interrogés sont des techniciens ayant obtenu une promotion sans changement d'activité. Chez les sous-traitants, ils ont des postes assortis de davantage de responsabilités, notamment managériales.

Les techniciens et agents de maîtrise ont également des activités de type administratif :

«Il faut aimer rester derrière l'écran à remplir des cases, on passe beaucoup de temps sur l'outil informatique à remplir des tableaux. D'ailleurs, le système informatique est complexe au niveau des applications. »

(Christelle, Aéro, baccalauréat, 35-45 ans) 
«Les aspects administratifs et bureaucratiques sont un peu lourds à force. Moi, je me sens plus "bureaucrate" que "technicien". »

(Christophe, Protométal, BTS, 35-45 ans)

Ces tâches nécessitent des compétences en informatique ; en particulier, techniciens et agents de maitrise se doivent d'avoir une solide connaissance du système utilisé par l'entreprise. Les applications qui le constituent sont parfois compliquées et pas toujours compatibles entre elles.

En outre, la maîtrise de l'anglais est désormais essentielle : « L'anglais est de plus en plus présent. Avant, les carnets d'entretien étaient disponibles en français mais ce n'est plus le cas maintenant » (Michael, Aéro, licence 3 Pro, 25-35 ans).

On demande également aux techniciens de faire preuve de polyvalence : «C'est jamais la même chose, il n'y a pas de routine, on travaille sur tous les appareils, au Japon, aux États-Unis, dans les autres services, etc. » (Michael, Aéro, licence 3 pro, 25-35 ans). Les techniciens travaillent donc sur plusieurs types de machines (peu sont affectés sur une machine unique) et exercent des activités diverses. Concrètement, la polyvalence signifie qu'ils doivent connaître différents systèmes complexes, savoir s'organiser et être autonomes, notamment lorsqu'ils doivent apprendre par eux-mêmes à se servir d'un nouvel équipement à partir de notices d'utilisation, d'informations qu'ils ont obtenues auprès des fabricants ou des organismes de normalisation du secteur.

Si les techniciens doivent être polyvalents, c'est parce qu'ils occupent une position de coordinateurs au carrefour de plusieurs services, y compris externes (cf. supra). En effet, leur place entre le bureau d'études, l'atelier (les compagnons), les sous-traitants et les clients est centrale :

«On est l'intermédiaire entre le bureau d'études et les compagnons, ainsi que les services administratifs. Ça arrive aussi qu'on soit en contact avec le client, par l'intermédiaire du commercial. Au final, on dépend de beaucoup de services, de circuits, il faut se démener. Les services autour ne sont pas adaptés au court terme comme nous : on est le vilain petit canard car on ne tient pas toujours nos délais... »

(Carole, Aéro, DUT, 25-35 ans)

D'ailleurs, chez le donneur d'ordre, la modularité implique que les techniciens et agents de maîtrise pilotent les sous-traitants, même s'il n'existe pas de relation hiérarchique formelle. Sans pour autant bénéficier de l'appui de leur encadrement ${ }^{13}$ et par la conception d'une instrumentation technique et contractuelle sophistiquée, ils sont chargés d'exercer directement des pressions, parfois fortes, pour que les soustraitants respectent les délais et les contraintes de qualité. Ils doivent aussi veiller à ce que ces derniers réduisent leurs coûts et leurs prix. Dès lors, le relationnel a une place déterminante : une étroite collaboration avec les différentes parties prenantes est indispensable afin d'assurer une diffusion de l'information optimale. Les techniciens doivent en outre être capables d'analyser et prévoir les besoins internes et externes,

13. La plupart des entretiens montrent que les relations de coopération avec l'encadrement direct sont distendues. 
d'en proposer une synthèse tant aux collaborateurs internes qu'externes et d'être réactifs lorsqu'ils évoluent :

«On a des relations en interne avec le service rechange, qui fournit les pièces aux clients extérieurs, et avec les compagnons, sur la partie "série". Ça fait partie de mon job, de harceler les gens pour avoir les pièces en temps et en heure. On fait beaucoup de relationnel, au-delà des aspects techniques. »

(Christelle, Aéro, bac, 35-45 ans)

Ainsi les techniciens sont amenés à devoir interpréter des situations si bien que le contenu cognitif de leur activité est renforcé. Dans la mesure où ils ont à jouer un rôle de traducteur et de transmetteur de savoirs, leur situation de travail se décloisonne, ce que leur encadrement ne peut percevoir sans une coopération étroite avec eux.

Le corollaire est que le métier de technicien est souvent considéré comme indéfini, d'autant que fréquemment, il n'existe pas de réelle fiche de poste. Les techniciens interrogés ne savent souvent ni ce que c'est ni s'ils en ont une :

« Le rôle de préparateur n'est pas assez cerné, c'est vague comme métier, c'est flou, on ne sait pas si c'est à nous de faire les choses et du coup, on nous en demande de plus en plus et on a l'impression parfois de faire le boulot des autres. »

(Alexandre, Aéro, licence 3 pro, moins de 25 ans)

Comme nous l'avons déjà dit, pour mener à bien leurs activités, les techniciens dépendent souvent beaucoup de celles d'autres services. Coordinateurs, responsables des prescriptions entre parties prenantes aux intérêts parfois différents, ils tendent à faire de la gestion de projet, et sont appréciés pour leur capacité à décloisonner l'organisation du travail entre les différents services. Leurs tâches se sont élargies et ils ont désormais des fonctions très autonomes et prescriptives vis-à-vis d'autres services, qu'ils apprennent ce faisant à écouter, alors même qu'ils n'ont pas de position hiérarchique légitimant cette relation. Plus précisément, leur autonomie concerne la conception de dispositifs de gestion mais aussi de transmission et de partage de connaissances (sur l'évolution de la réglementation par exemple). C'est parce que les professions intermédiaires ont une activité de conception qu'une régulation conjointe (REYNAUD, 1995) est possible entre, d'une part, les prescriptions des services méthodes, planning ordonnancement et études, et, d'autre part, celles provenant des agents de maîtrise experts en programmation et des techniciens en fabrication, notamment chez les sous-traitants. Si une telle autonomie est requise pour répondre aux exigences des postes occupés, elle est toutefois contrôlée par des tableaux de bord précis, des objectifs chiffrés, des normes de qualité et des délais à respecter ou à faire respecter. On peut parler d' «autonomie contrôlée » sur ce point, ce qui signifie que l'autorité dans la prescription du travail se relâche pour laisser place à la créativité de l'individu, mais que la performance de l'individu n'en est pas moins évaluée in fine (COURPASSON, 2000). Si elle est plutôt bien acceptée par les techniciens, ils attendent en retour qu'elle soit valorisée et qu'elle se traduise par un changement de qualification. Ainsi, ils aspirent à une progression de carrière dans des délais plus rapides que ceux offerts par 
l'entreprise. C'est particulièrement le cas pour les techniciens disposant d'une licence professionnelle ou d'un niveau bac +2 :

« J'aimerais faire l'école d'ingénieur X. Je ne suis pas obligée pour passer coordinateur, je peux aussi passer à l'ancienneté mais c'est beaucoup plus long. »

(Carole, Aéro, DUT, 25-35 ans)

« Je n'ai pas de sentiment de blocage dans mon parcours, c'est ouvert pour ceux qui sortent d'un Master, mais pour nous, il faut vraiment gratter. »

(Michael, Aéro, licence 3 pro, 25-35 ans)

Dans ces professions intermédiaires, les tensions observées chez le donneur d'ordre semblent également présentes dans les entreprises industrielles sous-traitantes. Les techniciens d'atelier, pilotes de MOCN tout comme les agents de maîtrise qui font de la programmation de haut niveau, exercent eux aussi des activités de conception dans lesquelles ils doivent faire preuve d'autonomie. Chez Usireva toutefois, certains agents de maîtrise sont confrontés à des problèmes de compétences d'ordre à la fois technique (car les échanges avec les bureaux d'étude vont croissant) et managérial (changements dans l'organisation du travail). En effet, ils font face à ce que les soustraitants appellent la « remontée en conception », c'est-à-dire au développement au sein des entreprises sous-traitantes d'activités de conception nécessitant un haut niveau de qualification, qui était auparavant l'apanage des donneurs d'ordre. Pour les personnels les plus âgés promus agents de maîtrise, ce ne sont pas les compétences techniques qui posent des difficultés puisqu'ils étaient ouvriers qualifiés auparavant, mais les nouvelles méthodes de gestion d'équipe mises en place par l'encadrement supérieur : alors qu'ils étaient habitués à des relations directes, en face-à-face, celles-ci sont de plus en plus informatisées (courriels, agendas partagés, etc.). Pour les jeunes agents de maîtrise, dont la pratique des nouvelles technologies est plus aboutie, les obstacles se situent davantage dans la construction d'une légitimité technique vis-à-vis des plus âgés et dans la gestion des jeunes entrants.

De manière générale, dans les entreprises étudiées, nous assistons donc à une complexification du travail. Dans le même temps, les besoins en main-d'œuvre deviennent de plus en plus spécifiques dans la mesure où les expertises construites par les techniciens et les agents de maîtrise ne sont pas des processus régulés dans un espace professionnel homogène. C'est pourquoi le donneur d'ordre est devenu centre agréé de formation et travaille en partenariat avec les entreprises d'intérim qui interviennent sur son site : ces dernières souhaitent former leurs personnels pour leur permettre de répondre aux critères de certification qu'Aéro impose. La remontée en conception que nous avons observée, à laquelle s'ajoute la sophistication des machines, a pour conséquence que l'activité de conception est de plus en plus importante et complexe ; elle exige toujours plus d'autonomie pour aller chercher, négocier, acquérir et transmettre des connaissances entre des fonctions qui restent cloisonnées. Nos observations s'inscrivent donc dans la continuité de celles effectuées lors de travaux plus anciens (EYRAUD et al., 1988). 


\section{Qualification, recrutement et formation}

Notre étude met en évidence qu'il existe des différences notables, relatives au niveau de qualification exigé des techniciens et des agents de maîtrise entre petites et grosses entreprises, notamment entre les sous-traitants et le donneur d'ordre.

Quoi qu'il en soit, globalement, le diplôme est présenté comme le principal critère retenu pour accéder à ces emplois. Les principaux parcours représentés parmi nos enquêtés sont tout d'abord les baccalauréats scientifiques ou anciennement sciences et technologies de l'industrie (STI) (microtechnique ou électrotechnique), suivis d'un DUT (génie mécanique ou maintenance industrielle le plus souvent) et complétés par une licence professionnelle de maintenance aéronautique. Le DUT peut aussi être remplacé par un BTS contrôle industriel et régulation automatique, par exemple. Certains techniciens et agents de maîtrise ont des parcours courts et sont titulaires de CAP dont les spécialités ne correspondent pas aux métiers de l'aéronautique (CAP plombier, CAP fraiseur-outilleur, etc.). Enfin, quelques parcours sont atypiques : bac pro agricole, diplôme d'aide chimiste, BTS assistant de direction, etc.

Cette hétérogénéité des diplômes est la même que celle observée dans l'industrie automobile (CADET, GUITTON, 2013) et correspond en partie à des différences de générations entre "recrutés ». Les nouveaux sont plutôt recrutés à un niveau bac +2 ou $\mathrm{bac}+3$, alors que les anciens pouvaient l'être avec un $\mathrm{BEP}^{14}$ ou un CAP.

Le niveau BTS/DUT, voire licence professionnelle ${ }^{15}$, est donc privilégié actuellement pour les recrutements sur des postes de techniciens ou d'agents de maîtrise dans l'industrie aéronautique. En effet, de nombreux responsables nous ont expliqué que les jeunes de niveau BEP-CAP sont plus difficiles à encadrer et auraient des difficultés à s'adapter aux exigences de l'entreprise et du poste, principalement car leur socle de connaissances serait trop restreint. Une telle réserve s'applique également aux niveaux bac pro et BTS : «On a eu des bac pro mais pas en dessous - dans les anciens ils ont des formations de CAP. On embauche en atelier des bac pro et des BTS, mais avec ça, ils savent rien » (DRH Usireva).

Les canaux mobilisés pour recruter des techniciens de l'industrie restent traditionnels : Pôle emploi, intérim, candidatures spontanées, bouche-à-oreille, Association pour la formation professionnelle des adultes (Afpa), stagiaires de l'alternance, contrat de professionnalisation (niveau BTS/DUT et licence principalement). Cependant, trouver des profils adaptés semble difficile :

« Pour notre offre, on a reçu une dizaine de CV [curriculum vitæ], dont des gens qui étaient boulangers. [...] On a des jeunes très motivés en stage ; c'est pour ça qu'on ne comprend pas, on a l'impression qu'ils sont démotivés après. Par exemple, ce jeune qui voulait faire le BTS [et qui] cherchait une école en alternance : l'école ne

14. Brevet d'études professionnelles.

15. Le niveau licence professionnelle n'est pas reconnu dans les grilles de classification des entreprises pour les techniciens de l'industrie qui sont donc intégrés et rémunérés comme des diplômés de niveau bac +2 . 
lui donne rien, on lui a dit que dans la région il n'y avait pas d'organisme pour faire la formation en alternance. Il y a un sérieux problème dans la formation au niveau des lycées !»

(Luc, Usireva, DUT, 25-35 ans)

«Le problème avec les jeunes diplômés de BTS, c'est qu'ils veulent travailler en bureau d'études et pas en atelier. Plus personne ne veut faire ce métier, et ce sont les jeunes en échec scolaire qui sont envoyés en formation mécanique [...], et comme le parc machine s'accroît, il y a une hausse des besoins de main-d'œuvre. Il y a formation des personnels en place pour travailler sur les nouvelles machines mais l'augmentation du parc demande tout de même plus d'opérateurs, un besoin accru au niveau des méthodes pour l'analyse technique et aussi pour le contrôle qualité... C'est pas facile de trouver ces ressources, il manque des opérateurs, on le ressent à tous les niveaux [...]. Les industriels ont aussi leur part de responsabilité avec le fait que dans l'évolution des machines, à un moment, on a pensé ne plus avoir besoin de techniciens et uniquement de presse-boutons, avec de l'autre côté des ingénieurs. Or c'est faux, aujourd'hui il manque ces compétences intermédiaires [...]. Toutes formations confondues, on n'apprend pas le fond du métier. »

(Christophe, Protométal, responsable programmation, BTS, 35-45 ans)

Globalement, les grandes entreprises, qui présentent un certain nombre d'avantages pour les candidats (intéressement, treizième mois, possibilités de mobilité en interne, etc.), trouveraient plus facilement les profils qu'elles recherchent que les petites entreprises et les sous-traitants. Comme ces derniers offrent peu de perspectives de carrière aux techniciens, ceux-ci se tournent vers le marché externe du travail et donc vers le principal donneur d'ordre, qui recrute essentiellement des techniciens expérimentés, soit par la voie du réseau de sous-traitance, soit par la voie de l'intérim. Par conséquent, le donneur d'ordre n'investit dans la formation en alternance pour les jeunes que pour une partie de ses besoins d'embauche. De plus, lorsqu'il en recrute, il sélectionne sur le volet les techniciens sortant de formation initiale dans la mesure où ils ne connaissent alors globalement pas le métier. De fait les employeurs tendent à privilégier ceux qui ont de l'expérience ou ceux qu'ils ont eux-mêmes formés. La forme de contrat la plus fréquente est le contrat à durée indéterminée (CDI), faisant suite la majeure partie du temps à une période d'alternance ou d'intérim, qui représente une sorte de «période d'essai ».

La plupart des techniciens interrogés indiquent ne pas avoir reçu de formation spécifique durant leur période d'intégration. Ils apprennent leur métier sur le tas, au contact de collègues plus expérimentés ou en consultant des manuels présentant les opérations techniques de base. Comme nous l'avons évoqué plus haut, au carrefour de la production et du partage des connaissances entre le bureau d'études et l'atelier de maintenance/préparation, les techniciens de l'industrie que nous avons rencontrés sont parfois confrontés à des incompréhensions entre corps de métiers ne partageant pas le même langage et donc à des situations d'incertitudes. L'organisation modulaire ayant pour conséquence que l'activité de conception n'est plus limitée au bureau d'étude 
mais est assurée par différents services dans et hors de l'entreprise, ce qui multiplie les échanges et leur complexité, les employeurs ont tendance à embaucher des techniciens à un niveau de diplôme plus élevé que par le passé. Parallèlement, les techniciens en poste demandent de plus en plus à avoir accès à la formation professionnelle continue. Or, dans la mesure où les besoins en formation correspondent à des savoirs inédits et très spécifiques liés au développement de la modularité, les responsables d'équipes ne trouvent parfois pas de formations adaptées dans les environs de l'établissement :

« Si demain je devais faire faire une formation à un technicien méthode, il n’y a pas d'offre de formation... On doit remettre les plans de formation, je ne trouve pas ce qui correspond et ce qui cadre avec mes gars ; je trouve telle ou telle méthode mais pas ce qui colle avec les attentes en interne. »

(Christophe, Protométal, responsable programmation, BTS, 35-45 ans)

Bien souvent, les techniciens ne reçoivent aucune formation longue durant leur carrière, mais se contentent de quelques demi-journées de formation «facteur humain », systèmes de radionavigation, avionique, etc. Les responsables d'équipes doivent alors faire face à une double pression : non seulement ils doivent transmettre des connaissances aux techniciens qu'ils encadrent mais ils doivent assumer leur propre charge de travail avec des tâches importantes de planification/coordination, y compris avec le donneur d'ordre.

D'ailleurs, dans les entreprises enquêtées, la validation des acquis de l'expérience (VAE) est très peu mobilisée et ce, en raison de deux freins majeurs : d'une part, la procédure de VAE elle-même est méconnue et la codification des savoirs et compétences qu'elle nécessite exigerait un temps considérable de la part de travailleurs qui n'ont pas l'habitude d'un tel exercice ; d'autre part, l'entreprise ne souhaite pas promouvoir ce type de démarche qualifiante, car elle estime ne pas pouvoir offrir les salaires en adéquation avec le niveau de qualification acquis. Elle préfère par conséquent valoriser et promouvoir des compétences selon son propre référentiel.

\section{Rémunération et carrières}

Nous avons observé que les anciennes règles de gestion des ressources humaines n'ont plus cours. Alors qu'auparavant les entreprises embauchaient des techniciens niveau CAP qui pouvaient bénéficier par la suite de promotions, elles recrutent désormais à un niveau plus élevé (licence professionnelle) et les promotions se font attendre. La reconfiguration industrielle semble donc s'être traduite par celle de la gestion des ressources humaines et des trajectoires des professions intermédiaires.

Les jeunes, en particulier ceux qui sont issus de licence, sont amenés à développer une expertise spécifique dans la mesure où, possédant un bagage théorique élevé, ils travaillent avec des anciens et doivent apprendre de ces derniers une partie du métier de façon informelle. En parallèle, les anciens apprennent grâce aux plus jeunes à se familiariser avec les nouvelles technologies notamment, ce qui donne lieu à une 
hybridation forte des savoirs. Ainsi, quel que soit le statut - technicien ou agent de maîtrise dans l'aéronautique - et le degré d'avancement dans la carrière, les attentes en termes de rémunération restent assez similaires. En revanche, tous n'ont pas les mêmes attentes en termes de déroulement de carrière : seuls ceux qui ont obtenu un DUT ou un BTS aspirent à devenir cadres ou à travailler dans des services autres que celui de la fabrication.

Concrètement, et contrairement à l'industrie automobile (CADET, GUITTON, 2013) où des parcours de carrière très structurés peuvent être proposés, en particulier pour les agents de maîtrise dans la fabrication, il n'existe pas de véritable gestion des carrières des techniciens et des agents de maîtrise dans les entreprises enquêtées. En effet, promouvoir un candidat d'un niveau DUT/BTS sur un poste au sein du bureau des méthodes reste difficile, car c'est plutôt un niveau ingénieur qui y est attendu. Le seul intérêt qu'a le bureau d'études ou des méthodes à recruter un technicien ou un agent de maîtrise est lié au fait qu'il connaît la fabrication et a effectué un passage au sein du service concerné. Finalement, les perspectives des techniciens et des agents de maîtrise restent proches de celles des ouvriers qualifiés devenus techniciens, qui n'accèdent pas à ce type de mobilité au niveau ingénieur ou cadre (VENEAU, 1995).

D'après les propos de certains DRH, les techniciens et agents de maitrise manqueraient de vision stratégique, de compétences en management et de recul vis-à-vis de leur position dans l'entreprise. Ceux que nous avons rencontrés nous ont expliqué au cours des entretiens avoir le sentiment que leurs possibilités d'évolution limitées traduisaient un manque de reconnaissance de leur travail. Le donneur d'ordre, par exemple, n'accorde aucune promotion à une personne qui reste en poste. De plus, même lorsqu'ils acceptent des missions spécifiques à l'étranger, la reconnaissance tant symbolique que du point de vue de la rémunération ou de la carrière n'est pas au rendez-vous, ce que certains regrettent :

« Je suis parti deux ans en mission aux États-Unis. J'ai formé des personnes dans la filiale, j'étais chargé notamment de l'entretien de la relation client, j'ai eu beaucoup de responsabilités et quand je suis revenu, rien. Je suis retourné à mon poste initial sans changement hormis une augmentation de 50 euros bruts. »

(Alexandre, Aéro, licence 3 pro, moins de 25 ans)

D'ailleurs, lorsqu'une promotion intervient, c'est pour répondre à un besoin de l'entreprise, besoin qui n'a pas nécessairement été anticipé. Le technicien ou agent de maîtrise qui bénéficie de cet avancement n'a donc pas suivi en amont une formation qui lui aurait permis de s'adapter à son nouveau poste. Le donneur d'ordre propose sinon trois possibilités d'évolution. La première est le passage au statut cadre à l'ancienneté (vers 40-50 ans) ; le technicien ou l'agent de maitrise abandonne alors toute fonction technique. Ceux qui le souhaitent peuvent également suivre la formation d'ingénieur dispensée par l'Institut des techniques d'ingénieur de l'industrie (ITII Paca), qui a un partenariat avec Aéro : pendant trois ans, en formation continue, ils assistent à des cours le vendredi et le samedi et ont des travaux personnels à faire. Enfin, ils peuvent parfois 
obtenir une mobilité horizontale, mais la concurrence est rude : «On est plusieurs milliers dans l'entreprise, c'est difficile de sortir du lot, on n'a pas trop les moyens de montrer qu'on est performants » (Alexandre, Aéro, licence 3 pro, moins de 25 ans).

Dans les entreprises enquêtées, la mobilité difficile ou délicate des techniciens vers des positions d'encadrement s'explique sans doute en partie par les tensions auxquelles cette catégorie est soumise et qui découlent de la manière dont l'encadrement exerce son autorité. Techniciens supérieurs et ingénieurs sont de plus parfois en concurrence. Les techniciens souffrent d'un déficit de légitimité en management, ce qui n'est pas le cas des ingénieurs qui sont susceptibles d'encadrer dès leur recrutement. En même temps, cette faible légitimité, se double d'un faible intérêt des techniciens pour le management, ce qui est à mettre en rapport avec un contexte organisationnel où domine une forme d'autorité qui relève plus du contrôle que de la coopération nécessaire à une bonne coordination. Les techniciens préfèrent par conséquent se consacrer aux activités de recherche d'informations et de mise en réseau plutôt qu' au management. Des agents de maîtrise leur emboîtent le pas en cherchant à sortir du rôle de "garde-chiourme » (Christophe, Protométal, BTS, 35-45 ans).

Les techniciens travaillant chez le donneur d'ordre n'ont toutefois pas envie de faire une mobilité externe. Ils n'ont pas intérêt à aller travailler chez un sous-traitant car ils perdraient des avantages tels que le treizième mois, l'intéressement, etc., caractéristiques des grandes entreprises. Chez les sous-traitants, qui sont des entreprises plus petites, les possibilités de mobilité verticale sont pourtant plus grandes : il y est relativement facile de devenir chef d'atelier par exemple. Alors qu'ils ont commencé au bas de l'échelle, les agents de maîtrise bénéficient de promotions qui interviennent sans nécessairement tenir compte de leur diplôme.

Les techniciens et agents de maîtrise de l'industrie qui ont eu un parcours « atypique » (c'est-à-dire ceux n'ayant pas le diplôme a priori requis pour leur poste), s'estiment globalement bien payés ${ }^{16}:$ " Aujourd'hui, je me sens bien, je suis payé à ma juste valeur pour moi et je servirai cette entreprise du mieux que je pourrai car ils ont su me donner ma chance » (Luc, Usireva, DUT, 25-35 ans).

À l'inverse, ceux qui ont une licence professionnelle adaptée à leur poste ont le sentiment de ne pas être assez rémunérés, en particulier au regard des pressions qu'ils subissent dans le cadre de leur travail et des responsabilités qu'ils endossent et qu'ils considèrent ne pas être reconnues à leur juste valeur :

« Je suis à 1870 euros bruts... J'estime que je devrais être payé 2000 euros bruts pour la responsabilité que représente le fait de mettre le tampon ${ }^{17}$. »

(Nicolas, Aéro, licence 3 pro, 25-35 ans.)

16. Le salaire moyen avec cinq ans d'ancienneté est d'environ 1500 euros nets

17. «Mettre le tampon » signifie ici donner formellement l'autorisation pour les essais en vol de l'aéronef après avoir fait des essais au sol. Cette autorisation implique une responsabilité extrême vis-à-vis de la sécurité du pilote. 
«Dans cinq ans je pourrai être promu en tant que responsable, mais je n'ai pas envie d'attendre, je passe un entretien avec un RH en Australie dans quelques jours. »

(Aurélien, Aéro, licence 3 pro, 25-35 ans)

Si les salaires qui nous ont été indiqués sont supérieurs dans les petites entreprises, les techniciens y font davantage d'heures et n'ont pas les avantages cités plus haut.

Chez le donneur d'ordre, le niveau bac +3 n'est pas reconnu, si bien que ceux qui détiennent un diplôme de ce type sont considérés comme des techniciens, et non comme des techniciens supérieurs, ce qui est source de frustration. En comparaison, les compagnons, qui ont un BEP ou un CAP, peuvent obtenir des certifications qui leur donnent droit à des primes. Ils touchent en outre des rémunérations complémentaires diverses (déplacement, nuit, etc.). Certains compagnons gagnent donc davantage que des techniciens ayant une licence professionnelle.

Globalement, le sentiment de non-reconnaissance est prédominant puisque le niveau des rémunérations ne répond pas aux attentes :

«Au niveau du salaire, on a des augmentations de 5 à $6 \%$. Donc pour des techniciens, c'est de l'ordre de 50 euros bruts par mois, ça génère de la démotivation. »

(Christelle, Aéro, bac, 35-45 ans)

« Mon contrat est très mal rémunéré à cause du manque d'expérience : on me propose un salaire minimum, le $\mathrm{Smic}^{18}$, alors que j'ai $50 \mathrm{~km}$ à parcourir pour travailler. »

(Jean, Usireva, CAP, 25-35 ans)

De plus, les modes de reconnaissance salariale ne sont pas institutionnalisés et dépendent fortement du bon vouloir de l'encadrement direct : « Je n'ai pas de sentiment de reconnaissance, ça dépend beaucoup du chef que l'on a » (Carole, Aéro, DUT, 25-35 ans).

Compte tenu de la position stratégique des techniciens dans les processus d'innovation et de normalisation, la hiérarchie a recours à l'intéressement et aux primes pour faire évoluer leurs salaires, espérant ainsi éviter les demandes de mobilité. Pour autant, avec le sentiment d'être mal payés et au bas de l'échelle, les techniciens étudiés témoignent, y compris chez le donneur d'ordre, d'une attitude de désaffiliation visà-vis de leur entreprise. Ils sont en attente d'une gestion des ressources humaines qui soit plus attentive à leur parcours et leur accorde une rémunération à la hauteur de leurs efforts et des responsabilités qu'ils assument : «L'entretien annuel, je l'ai passé une fois en trois ans. Ça fait deux ans que je demande à le passer, mais rien à faire ; du coup on relance par mail » (Michael, Aéro, licence 3 pro, 25-35 ans).

18. Salaire minimum interprofessionnel de croissance. 
Dans cet article, notre objectif était d'observer dans quelle mesure les changements d'organisation dans le secteur aéronautique ont eu des répercussions sur les professions intermédiaires. Dans une industrie où la modularité implique la sous-traitance de sous-systèmes entiers, techniciens et agents de maîtrise sont désormais mobilisés pour assurer un rôle de médiation via la production et le partage de connaissances dans des activités de conception collective étendues à l'ensemble de la chaîne de valeur : conception à l'interface entre les bureaux d'études/méthodes, recherche de solutions en fabrication sous-traitante, conception d'outils numériques de gestion de la soustraitance, conception de dispositifs d'animation, de maintenance et de formation en interne ou dans les réseaux clients.

Les profils des techniciens sont donc de plus en plus divers, ce qui brouille la compréhension de leur identité. Dans la grande entreprise donneur d'ordre que nous avons enquêtée, ils passent de l'atelier aux services de planification, de gestion, de contrôle, et d'animation-formation technique. Dans les plus petites entreprises soustraitantes, ils jouent de plus en plus un rôle d'interface entre les bureaux d'études, le service commercial et celui de la fabrication, en raison d'une remontée en compétence de conception et de commercialisation d'une partie de ces sous-traitants, qui cherchent à s'autonomiser de leurs donneurs d'ordres. Par conséquent, appartenir aux professions intermédiaires n'a ni le même sens ni les mêmes implications que l'on travaille chez le donneur d'ordre ou chez ses sous-traitants.

Toutefois, dans les deux types d'entreprises, leurs tâches impliquent des activités de coordination telles que celles qu' exercent les cadres. Or ces derniers savent de moins en moins prescrire le travail aux techniciens et aux agents de maîtrise, car les savoirs qu'ils apprennent dans leur activité, s'ils produisent des résultats visibles, restent peu formalisés. La mobilité externe de ces professions intermédiaires dans la quête d'un meilleur statut pourrait entraîner des pertes de savoirs spécifiques au détriment de l'entreprise, savoir que les cadres ne peuvent transmettre aux nouvelles recrues puisqu'ils n'y ont pas eu accès.

\section{BibLIOGRAPHIE}

Acha V., Brusoni S. (2008), "The Changing Governance of Knowledge in Avionics", Economics of Innovation and New Technology, vol. 17, $\mathrm{n}^{\circ}$ 1-2, pp. 43-59.

Araujo L. (2006), "Modularity, Systems Integration and Supply Chain Leadership", 4th Worldwide Symposium on Purchasing and Supply Chain Management, University of San Diego (Californie).

Bercot R., Capdevielle P., Héran F., Hillau B., Lhotel H., Mouy P., Peyrard C., Sueur M., ZaRIFIAN P. (1988), « Ouvriers qualifiés, maîtrise et techniciens de production dans les industries en cours d'automatisation »,Études, ${ }^{\circ} 43$, volume I : synthèse générale, Marseille, Centre d'études et de recherches sur les qualifications (Céreq). 
Bonnafos (DE) G. (1989), « Le comportement des techniciens au travail : l'interaction entre les projets individuels et les pratiques de gestion du personnel », Bref, n ${ }^{\circ} 43$, Marseille, Céreq. Bosc S. (2008), Sociologie des classes moyennes, Paris, La Découverte.

Bouffartigue P. (1994), «La socialisation professionnelle des jeunes BTS-DUT. Entre diplôme et statut, des identités incertaines », Formation emploi, $\mathrm{n}^{\circ}$ 45, pp. 3-23.

Bouffartigue P., GAdÉA C. (1996), « Un héritage à l'épreuve. Bref panorama des évolutions dans la formation et l'emploi des ingénieurs en France », Formation emploi, n ${ }^{\circ}$ 53, pp. 5-13.

Brusoni S., Prencipe A., Pavitt K. (2001), “Knowledge Specialization, Organizational Coupling and the Boundaries of the Firm: Why Firms Know More than they Make?", Administrative Science Quarterly, vol. 46, n 4, pp. 597-621.

Buisson M.-L., GAdille M. (2013), «Les techniciens dans l'aéronautique : une position ambiguë face au déconfinement des situations de travail », in Cadet J.-P., Guitton C., Les Professions intermédiaires. Des métiers d'interface au cœur de l'entreprise, Paris, Armand Colin, pp. 165-174.

CADEt J.-P., Guitton C. (dir.) (2013), Les Professions intermédiaires. Des métiers d'interface au cour de l'entreprise, Paris, Armand Colin.

Carré P., Moisan A., Poisson D. (1997), L’Autoformation. Psychopédagogie, ingénierie, sociologie, Paris, Presses universitaires de France.

CourPasson D. (2000), L'Action contrainte : organisations libérales et domination, Paris, Presses universitaires de France.

Deauvieau J., Dumoulin C. (2010), « La mobilité socioprofessionnelle des professions intermédiaires : fluidité, promotion et déclassement », Économie et statistique, n 431-432, pp. 57-72.

Eyraud F., Iribarne (D’) A., Maurice M. (1988), « Des entreprises face aux technologies flexibles : une analyse de la dynamique du changement », Sociologie du travail, vol. 30, $\mathrm{n}^{\circ} 1$, pp. 55-77.

Frigant V., TALвOT D. (2004), « Convergence et diversité du passage à la production modulaire dans l'aéronautique et l'automobile en Europe », Actes du Gerpisa, n 37, pp. 107-118.

GAdille M., MACHADO J. (2010), « La formation dans les PME d'un pôle : rapports au savoir et division du travail », Éducation permanente, n ${ }^{\circ} 182$, pp. 107-121.

Gadille M., Machado J. (2012), "Multilevel Effects of a Method of Expert's Knowledge Transfer", VINE - Journal of Information and Knowledge Management Systems, vol. 42, n⿳ 3-4, pp. 350-364.

GÉLOT D. (2006), « Le rôle de l'encadrement intermédiaire dans la formation en entreprise », Travail et Emploi, $\mathrm{n}^{\circ} 107$, pp. 47-58.

HAAs J. (2010), «PME aéronautiques : l'enjeu des fonctions "support" », Éducation permanente, $\mathrm{n}^{\mathrm{o}} 182$, pp. 92-102. 
Hatchuel A. (1996), « Coopération et conception collective. Variété et crises des rapports de prescription », in Terssac (de) G., Friedberg E. (dir.), Coopération et conception, Toulouse, Octarès, pp. 101-121.

Hatchuel A., Le Masson P., Weil B. (2002), « De la gestion des connaissances aux organisations orientées conception », Revue internationale des sciences sociales, $\mathrm{n}^{\circ} 171$, pp. 29-42.

Jobert A., TAllard M. (1992), « Système de classification et structuration de la catégorie des techniciens », Sociétés contemporaines, nº 9, pp. 143-158.

LePlat J. (1988), «Les habiletés cognitives dans le travail », in Perruchet P. (dir.), Les Automatismes cognitifs, Liège, P. Mardaga, pp. 139-172.

Maurice M., Sellier F., Silvestre J.-J. (1982), Politique d'éducation et organisation industrielle en France et en Allemagne : essai d'analyse sociétale, Paris, Presses universitaires de France.

Maurice M., Sorge A. (1989), « Dynamique industrielle et capacité d'innovation de l'industrie de la machine-outil en France et en RFA », Document Lest, $\mathrm{n}^{\circ}$ 89-1.

MöBus M., DelanoËA. (collab.) (2009), « Les professions intermédiaires dans les entreprises. Techniciens, agents de maîtrise et assimilés : cadrage statistique », Nef, ${ }^{\circ} 39$, Marseille, Céreq.

Möвus M., DelanoË A. (2013), « Les professions intermédiaires des entreprises : la nébuleuse en chiffres », in Cadet J.-P., Guitton C. (dir.), Les Professions intermédiaires. Des métiers d'interface au cour de l'entreprise, Paris, Armand Colin, pp. 29-46.

Reynaud J.-D. (1995), Le Conflit, la Négociation et la Règle, Toulouse, Octarès.

Romelaer P. (2005), «L'entretien de recherche », in Roussel P., Wacheux F., Management des ressources humaines : méthodes de recherche en sciences humaines et sociales, Bruxelles, De Boeck, pp. 101-134.

Terssac (DE) G., Chabaud C. (1990), « Référentiel opératif commun et fiabilité », in Leplat J., Terssac (de) G. (dir.), Les Facteurs humains de la fiabilité dans les systèmes complexes, Marseille, Octarès-Entreprises, pp. 111-139.

Trouvé P. (1997), Les Agents de maîtrise à l'épreuve de la modernisation industrielle : essai de sociologie d'un groupe professionnel, Paris, Montréal, L'Harmattan.

VENEAU P. (1995), «Évolution de la mobilité et accès à la position de technicien », Sociétés contemporaines, $\mathrm{n}^{\mathrm{O}}$ 22-23, pp. 185-206.

WitToRSKi R. (1998), « De la fabrication des compétences », Éducation permanente, $\mathrm{n}^{\circ} 135$, pp. $57-70$. 